\title{
Critical comparison of energy management algorithms for lithium-ion batteries in renewable power plants
}

\author{
Alberto Berrueta, Adrián Soto, Miguel García, Íñigo de la Parra, Pablo Sanchis, Alfredo Ursúa Department of \\ Electrical, Electronic and Communication Engineering \\ Institute of Smart Cities, Public University of Navarre \\ Pamplona, Spain \\ alberto.berrueta@unavarra.es
}

\begin{abstract}
Lithium-ion batteries are gaining importance for a variety of applications due to their price decrease and characteristics improvement. A good energy management strategy is required in order to increase the profitability of an energy system using a Li-ion battery for storage. The vast number of management algorithms that has been proposed to optimize the achieved profit, with diverse computational power requirements and using models with different complexity, raise doubts about the suitability of an algorithm and the required computation power for a particular application. The performance of three energy management algorithms based on linear, quadratic, and dynamic programming are compared in this work. A realistic scenario of a mediumsized PV plant with a constraint of peak shaving is used for this comparison. The results achieved by the three algorithms are compared and the grounds of the differences are analyzed. Among the three compared algorithms, the quadratic one seems to be the most suitable for renewable-energy applications, given the undue simplification of the battery aging required by the linear algorithm and the discretization and computational power required by a dynamic algorithm.
\end{abstract}

\section{Keywords}

Lithium-ion battery, energy management, renewable energy, microgrid

\section{INTRODUCTION}

Due to environmental and economical issues, renewable power plants are thriving around the World. Significant efforts are dedicated to increase the renewable energy share in the electricity grid. Energy management is a key issue that concerns important aspects of energy storage systems (ESSs), such as their sizing, management, degradation and profitability. Among the available ESSs, lithium-ion batteries are an attractive option, given their outstanding power capability, high energy density and decreasing price [1].

The energy management strategy of a Li-ion battery has a direct impact on the system profitability, given that this is the algorithm that governs the energy dispatch to the electricity grid. On the one hand, a dispatch during the time with highest electricity price leads to higher economic income. However, the aging phenomena of the battery depend on its power flow [2]. Therefore, the management strategy should be calculated in order to enlarge the battery lifetime. Given the increasing number of applications in which a management strategy is required, this is currently a hot research topic. The selection of an energy management algorithm should depend on the particular installation that needs to be governed, and should take into account parameters such as the computation power, the battery size and the required accuracy.

The aim of this contribution is to analyze and compare various algorithms commonly used for the computing of the energy management of a Li-ion battery. Their specific characteristics and the achieved result determine the most suitable algorithm to be used in each particular setup or application. In order to make a realistic comparison, a scenario based on a renewable energy power plant is used for the computation of the energy management of the ESS, as detailed in Section II. The three studied algorithms are detailed in Section III. The first of them is based on dynamic programming, a powerful tool that deals with non-linear problems, but with high computational requirements. The second algorithm is based on linear programming, which requires a simple, linear model and a noticeably lower computational power. The third one is a quadratic-programming algorithm, which tries to find the trade-of between accuracy and simplicity. The comparison between them is detailed in Section IV, in which the three algorithms are used to compute the management strategy for the ESS in ten problems with various battery sizes, the achieved revenue is calculated for each of them and the results are contrasted. Finally, Section V summarizes the main conclusions of the contribution.

We would like to acknowledge the support of the Spanish State Research Agency (AEI) and FEDER-UE under grants DPI2016-80641-R and DPI201680642-R and of Government of Navarra through research projects PI020 RENEWABLE-STORAGE and 0011-1411-2018-000029 GERA. 


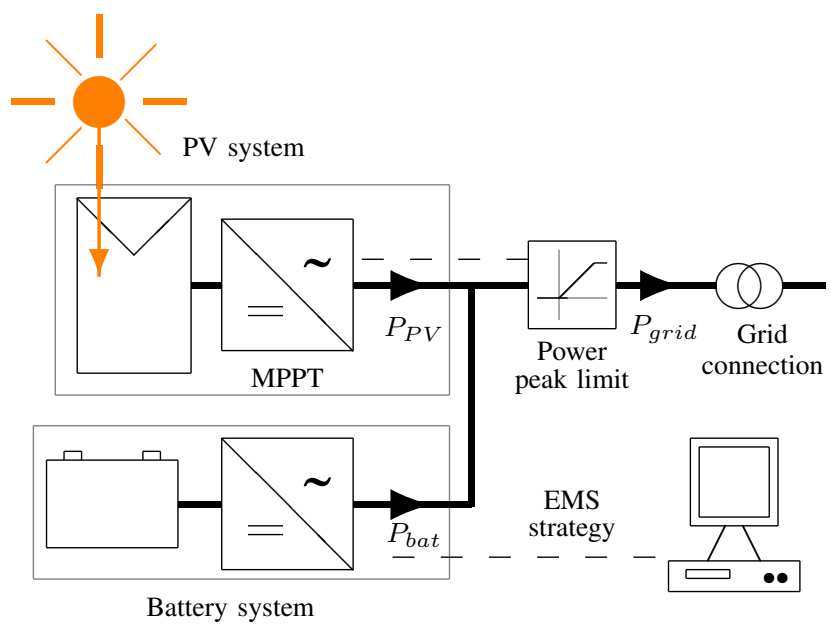

Fig. 1. System diagram of the PV+ plant.

Table I

MAIN TECHNICAL SPECIFICATIONS OF THE CASE STUDY.

\begin{tabular}{ll}
\hline Characteristic & Value \\
\hline PV panel & Yingli Solar YL250P-29b \\
PV peak power & $117.5 \mathrm{~kW}$ \\
PV inverter & Ingeteam IS 3PLAY \\
PV inverter rated power & $100 \mathrm{~kW}$ \\
Battery converter & FeCon BAT50 \\
Battery converter power & $50 \mathrm{~kW}$ \\
Feed in limitation & $60 \%$ of the inverter power \\
Grid voltage & $13.2 \mathrm{kV}$ \\
Annual solar radiation & $1,700 \mathrm{kWh} \mathrm{m}^{-2}$ \\
\hline
\end{tabular}

\section{THE ENERGY MANAGEMENT PROBLEM: A PV-BATTERY PLANT WITH PEAK SHAVING LIMITATION}

A realistic case study is proposed in this contribution in order to accomplish the comparison between the energy management strategies. It consists of a medium-sized PV plant with a peak power of $100 \mathrm{~kW}$ located in an industrial estate of Navarra, in the north of Spain. The following subsections summarize the main data of the PV system and the Li-ion battery.

\section{A. PV system}

As shown in Figure 1, the PV system comprises the PV array and the PV inverter. The PV array is formed by the connection of $n_{\text {pan }}=470 \mathrm{PV}$ panels, each one with a peak power of $P_{\text {pan }}=250 \mathrm{Wp}$. Therefore, the peak power of the PV array is $P_{\text {peak }}=117.5 \mathrm{kWp}$. It is a common design strategy to slightly oversize the PV field compared to the inverter. In this case, $P_{P V, \text { inv }}=0.85 \cdot P_{\text {peak }} \approx 100 \mathrm{~kW}$.

The control algorithm of the PV inverter is the maximum power point tracker (MPPT). In a normal situation, the MPPT achieves maximum PV power $\left(P_{P V}=P_{P V}^{*}\right)$. However, as the saturation of the distribution network is a concern in industrial estates, a feed-in power limitation is considered. As an interesting limit proposed in various countries, the selected value is $60 \%$ of the inverter nominal power. Therefore, when a power higher than the maximum feed-in power $\left(P_{\text {grid,max }}\right)$ can be injected into the grid, the inverter power is limited, as shown in Figure 1 and the extra available power is not generated $\left(P_{P V} \leq P_{P V}^{*}\right) . P_{\text {grid }}$ is assumed to be unidirectional from the plant to the grid, since regulations in most countries do not permit the supply of grid power to the generation plant. Measured meteorological data in Spain are taken from the free-access database Meteonavarra [3]. The mentioned technical characteristics of the case study are summarized in Table I.

\section{B. Li-ion battery}

In order to model the battery electrical performance, an equivalent circuit consisting of a voltage source dependent of the state of charge $V_{O C}(S O C)$ and an internal series resistance $R_{i}$ is used [4]. This model predicts the battery efficiency with high accuracy, given that it takes into account its current and SOC dependencies. The open circuit voltage $v_{O C}$ is related to the $S O C$, as proposed by previous research papers [5], [6]. This characteristic curve remains virtually constant for the whole battery lifetime [7] and is included in the model as a lookup table. The internal resistance $R_{i}$ also depends on the SOC, and the expression proposed in previous studies is used to model this influence [6]. As the battery ages, this parameter is scaled up, as detailed below. The SOC defines the stored capacity relative to the actual full capacity $(C(S O H))$ :

$$
S O C(t)=S O C\left(t_{0}\right)-\int_{t_{0}}^{t} \frac{i_{b a t}(t)}{C(S O H)} d t
$$


Table II

BATTERY AGING MODEL PARAMETERS FOR $\Delta C$ (FIRST COLUMN) AND $\Delta R_{i}$ (SECOND COLUMN), FROM [6].

\begin{tabular}{|c|c|c|c|c|}
\hline & Parameter & Unit & $\Delta C$ & $\Delta R_{i}$ \\
\hline \multirow{3}{*}{$\frac{\vec{d}}{\frac{\vec{d}}{\widetilde{U}}}$} & $a_{v}$ & - & $2.716 \cdot 10^{5}$ & $9.486 \cdot 10^{3}$ \\
\hline & $a_{0}$ & V & 3.1482 & 3.096 \\
\hline & $a_{T}$ & K & 6976 & 5986 \\
\hline \multirow{6}{*}{ 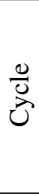 } & $b_{0}$ & - & $2.71 \cdot 10^{-5}$ & $2.28 \cdot 10^{-5}$ \\
\hline & $b_{v}$ & $\mathrm{~V}^{-1}$ & $3.14 \cdot 10^{-4}$ & $3.208 \cdot 10^{-4}$ \\
\hline & $b_{v 0}$ & $\mathrm{~V}$ & 3.683 & 3.741 \\
\hline & $b_{D O D}$ & - & $1.61 \cdot 10^{-6}$ & $3.404 \cdot 10^{-6}$ \\
\hline & $b_{I}$ & - & $1.56 \cdot 10^{-5}$ & $1.56 \cdot 10^{-5}$ \\
\hline & $b_{\exp }$ & $\mathrm{h}$ & 1.8 & 1.8 \\
\hline
\end{tabular}

Note that the negative sign before the integral term is due to the sign of the current $i_{b a t}$ defined as battery discharging current to be consistent with the sign of $P_{b a t}$.

The battery aging has a capital importance for the energy management strategy, given that the aging phenomena are determined by the battery use. As commonly done with Li-ion batteries, its aging effects are divided into calendar and cycle aging, which are assumed to be independent of each other [8]. A linear time dependency of capacity fade and resistance rise is assumed for the calendar aging, as shown in Equation 2 and Equation 3. For the cycle aging modeling, the dependence of capacity fade and impedance rise with the number of equivalent cycles $Q$ is assumed to be also linear:

$$
\begin{gathered}
\frac{\Delta C(t, Q)}{C(t)}=-\left(\alpha_{C} \cdot t+\beta_{C} \cdot Q\right) \\
\frac{\Delta R_{i}(t, Q)}{R_{i}(t)}=\alpha_{R_{i}} \cdot t+\beta_{R_{i}} \cdot Q
\end{gathered}
$$

where $t$ is expressed in years and $Q$ in equivalent full cycles.

Parameters $\alpha_{j}$ (j representing $C$ and $R_{i}$ ) determine the calendar aging, while $\beta_{j}$ govern the cycle aging. The dependencies of these four parameters are expressed, as proposed in [6], by the following equations:

$$
\begin{aligned}
\alpha_{j} & =a_{v, j} \cdot\left(v_{\text {cell }}-a_{0, j}\right) \cdot \exp \left(-\frac{a_{T, j}}{T}\right) \\
\beta_{i} & =b_{0, j}+b_{v, j} \cdot\left(v_{c y c}-b_{v 0, j}\right) \cdot{ }^{2}+b_{D O D, j} \cdot D O D \\
& +b_{I, j} \cdot \exp \left(b_{\exp } \cdot \frac{\left|i_{\text {bat }}\right|}{C}\right)
\end{aligned}
$$

Given that Li-ion batteries require the operating temperature to be controlled, the battery temperature is assumed to be constant for this case study, being $T=30^{\circ} \mathrm{C}$ in Equation 4. The aging model parameters, as proposed in [6], are shown in Table II.

Considering that the battery lifetime is over when its capacity fade or impedance rise reach $20 \%$, of the rated values, $\triangle S O H$ is defined as follows:

$$
\Delta S O H=-\frac{1}{0.2} \cdot \max \left[\left|\frac{\Delta C}{C}\right|,\left|\frac{\Delta R_{i}}{R_{i}}\right|\right]
$$

\section{Economical scenario and objective function}

The energy management strategies are designed to maximize the revenue of the PV-battery system. Therefore, the objective function $J$ is an economic revenue obtained by the inclusion of a battery in the PV plant. This revenue comes from the combined effect of the augmented PV energy injected into the grid and the displacement of the PV generation to the time interval with highest energy price. The battery aging costs are accounted by taking battery aging into account. This function takes shape into the following equation:

$$
\begin{gathered}
J=\int_{t_{0}}^{t}\left[\left(P_{\text {grid }, \mathrm{PV}-\text { bat }}-P_{\text {grid }, \mathrm{PV}}\right) \cdot P C_{\text {elec }}+\right. \\
\left.\Delta S O H \cdot P C_{\text {bat }}\right] d t
\end{gathered}
$$

The economic variables involved in this expression are $P C_{e l e c}$, which is the price of electricity (in EUR per kWh), and $P C_{b a t}$, which is the purchase price of the Li-ion battery (in EUR).

It is interesting to make the analysis under realistic, variable electricity prices. However, some grid services are not properly remunerated, based on current market rules. Therefore, the real electricity price in Spain is scaled up to an average price of EUR 0.14 per kWh maintaining the current variability of market prices. A price of EUR 250 per kWh for the battery system is considered, which includes the cost of the battery bidirectional power converter. 

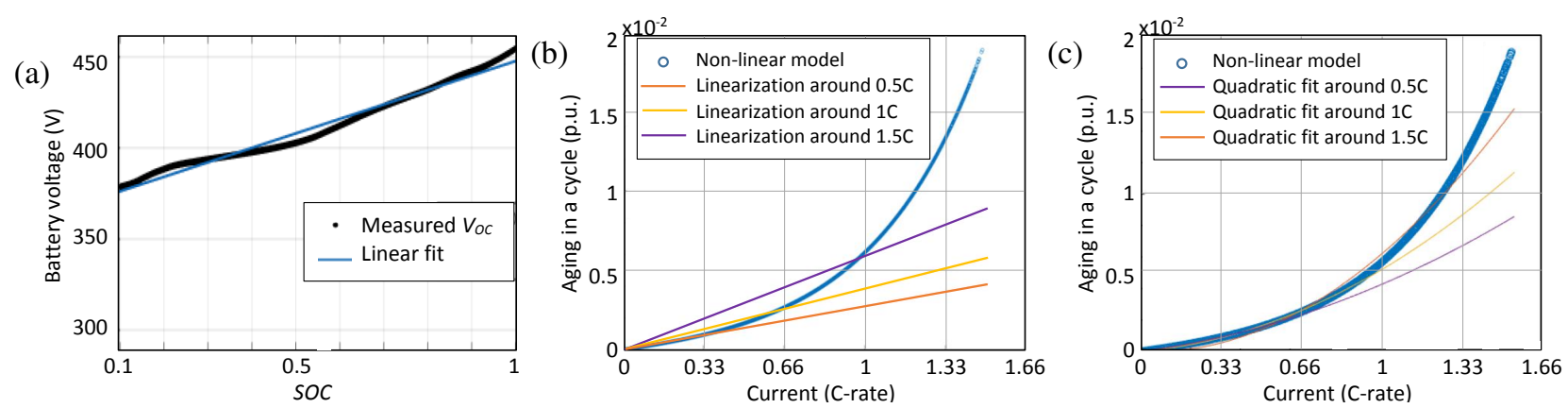

Fig. 2. Comparison between the non-linear model and the fittings of the linear and quadratic models: (a) linear fit of $V_{O C}(S O C)$, (b) linear fit of cycle aging ( $\beta_{i}$ in Equation 5), and (c) quadratic fit of cycle aging ( $\beta_{i}$ in Equation 5).

\section{ENERGY MANAGEMENT STRATEGIES FOR LI-ION BATTERIES}

Three of the most common algorithms used by energy management systems (EMSs) are compared in this contribution. Their main characteristics are explained in this section. Besides their features, the required adaptations of the management problem, such as simplification or linearization of some expressions, are compiled in the subsequent subsections.

\section{A. Dynamic programming}

The dynamic programming tackles a complex, sequential optimization problem by breaking it down into several simpler subproblems. The main problem is solved thanks to the Bellman Principle of Optimality, which states that a decision subsequence of the optimal decision sequence is also an optimal solution of the corresponding subproblem among the subsequences with the same final state [9].

The dynamic programming is a suitable algorithm for EMSs, given the sequential and non-linear nature of these problems. However, its main drawbacks are the high computational requirements and the exponential increase of the problem complexity entailed by an increasing number of variables. In a problem such as the one studied in this contribution, with a temporal frame imposed by the solar radiation (one day), the number of variables limit the minimum time step that can be tackled. As a trade-off between reasonable computing time and results accuracy, a time step of one hour is chosen.

The algorithm uses the model and objective function detailed above, along with the following operational constraints:

$$
\begin{aligned}
v_{\text {bat }, \text { min }} \leq v_{\text {bat }} & \leq v_{\text {bat }, \text { max }} \\
S O C_{\text {min }} \leq S O C & \leq S O C_{\text {max }} \\
i_{\text {bat }, \text { min }} \leq i_{\text {bat }} & \leq i_{\text {bat }, \text { max }} \\
P_{\text {grid,min }} \leq P_{\text {grid }} & \leq P_{\text {grid }}^{*} \\
P_{P V} & \leq P_{P V}^{*} \\
P_{b a t} & \leq P_{N, \text { conv }}
\end{aligned}
$$

\section{B. Linear programming}

A lineal algorithm finds the optimal value of a linear function, subject to linear equality and inequality constraints. Its main advantage is the reduced computational requirement. However, the linear fits required by this technique do not allow the use of non-linear models or constraints. This issue entails concerns about the loss of accuracy of linear algorithms.

Specifically, three expressions of the model explained above need to be modified in order to use a linear algorithm:

- The relationship $V_{O C}(S O C)$ cannot be represented by measured data stored in a lookup table. By contrast, a linear expression needs to be fitted. Figure 2 (a) shows this fit, which provides a value of $R^{2}=0.977$.

- The efficiency of the battery cannot be modeled by the internal resistance, $R_{i}$, since a resistance implies power losses proportional to the square of the current: $P_{l o s s}=R_{i} \cdot I_{b a t}^{2}$. For a linear algorithm to be applied, the power losses need to be considered as a constant value.

- The cycle aging term $\beta_{i}$ (see Equation 5) has an exponential term, which needs to be linearized. As represented in Figure 2 (b), a linear expression cannot properly fit this exponential trend of the cycle aging for a wide current range. Therefore, the linearization needs to be performed by taking the battery current into account. Three linear fits are shown in this figure, considering battery current rates below $0.5 \mathrm{C}$, below $1 \mathrm{C}$ and below $1.5 \mathrm{C}$. Given that the time step considered in this contribution is $1 \mathrm{~h}$, the maximum battery current that can be calculated is $1 \mathrm{C}$. Therefore, this is the chosen fit, which provides a value of $R^{2}=0.90$. The actual implication of this linearization is that the dependency of cycle aging on the battery power cannot be taken into account by a linear model, as detailed below. 


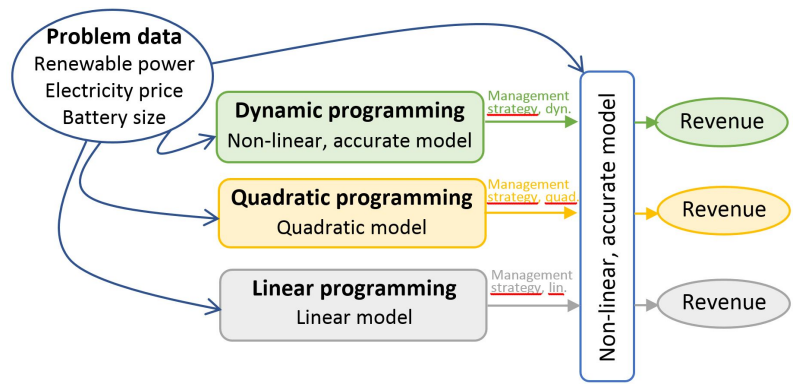

Fig. 3. Flow diagram of the comparison between the three algorithms, represented in different colors, based on uniform database and revenue calculation.

\section{Quadratic programming}

A quadratic optimization algorithm deals with quadratic objective functions, even though the constraints need to be linear. Given that the constraints of the studied problem are already linear equations, this algorithm requires lower simplifications of the model than a linear algorithm. Specifically, an internal resistance $R_{i}$ can be modeled by means of a quadratic model, even though its dependency with SOC cannot be taken into account. This improves the simplification of a constant battery efficiency, including its dependency on the managed power. Another improvement of a quadratic objective function, compared to a linear one, is the inclusion of the dependency of cycle aging (parameter $\beta_{i}$ of Equation 5) on battery current. Additionally, the relationship $V_{O C}(S O C)$ shown in Figure 2 (a) can be fitted by a second-order polynomial expression, as shown in Figure 2 (c). These three improvements make the quadratic model more realistic than a linear one.

Among these three enhancements, the most relevant one is the aging modeling, which can be seen by comparing Figure 2 (b) and (c). The coefficient of determination of these two fits for currents lower than $1 \mathrm{C}$ are $R_{\text {linear }}^{2}=0.90$ and $R_{\text {quadratic }}^{2}=0.99$.

\section{PERFormance COMPARISON OF ENERGy MANAGEMENT STRATEgIES}

We present in this section a comparison between the algorithms explained above, used to calculate the energy management of a Li-ion battery. Special attention is devoted to:

- Computation requirements.

- Applied battery model (the most accurate available, its linear fit, or the quadratic fit).

- Computed management strategy and achieved revenue.

- Problem characteristics such as time step and maximum battery power.

A flow diagram of this comparison is shown in Figure 3. The input data for the optimization problem are the renewable power generated by the plant during a whole year, the hourly price offered in the electricity market and the battery size. The three algorithms explained in the previous section, along with the appropriate model, are used to calculate three different management strategies for the year. As explained above, the most accurate model available for the system has a number of non-linear relationships. Therefore, it needs to be simplified in order to be used by the quadratic and linear algorithms. The economic income and aging of the battery after the year of operation following the three management strategies are calculated by means of the non-linear, accurate model. Finally, the revenue obtained by each algorithm is computed by the subtraction of the battery aging cost from the economic input achieved by selling electricity. For better comparison, the revenue is provided as relative values, normalized with the revenue obtained by the dynamic algorithm as follows:

$$
\text { Relative revenue }=\frac{\text { Studied revenue }}{\text { Dynamic algorithm revenue }}
$$

Given that the ratio between the battery and the plant size determine the variable discretization required for the dynamic algorithm, ten problems with battery sizes ranging from $0.10 \mathrm{kWh} / \mathrm{kWp}$ to $2.6 \mathrm{kWh} / \mathrm{kWp}$ are solved using each algorithm. The battery size is defined as follows:

$$
\mathrm{kWh} / \mathrm{kWp}=\frac{\text { Battery capacity }(\mathrm{kWh})}{\mathrm{PV} \text { peak power }(\mathrm{kWp})}
$$

For the sake of comparability, the optimization problem in all the cases is reduced to one day, which requires the imposition of a battery SOC at the beginning and at the end of each day. Then, 365 problems are solved in order to compute the management strategy for the whole year.

The comparison between the dynamic and the linear algorithms is shown in Figure 4. Figure 4 (a) shows the battery state of charge. Even though both algorithms calculate similar strategies, based on a battery charge using a surplus of PV power and battery discharge during the evening, there is an interesting difference. While the dynamic algorithm schedules a soft battery discharge during the whole evening, even though the electricity price has slight variations, the linear algorithm schedules a high-current battery discharge during the hour with highest electricity price. This is due to the linear fit of the aging model, 
(a)

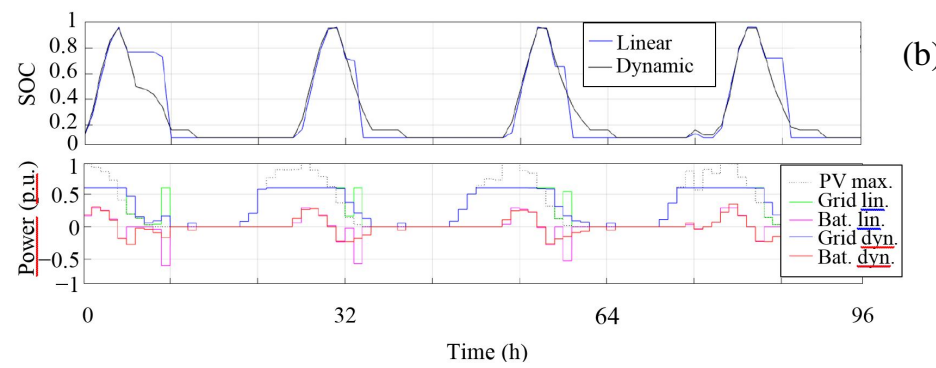

(b)

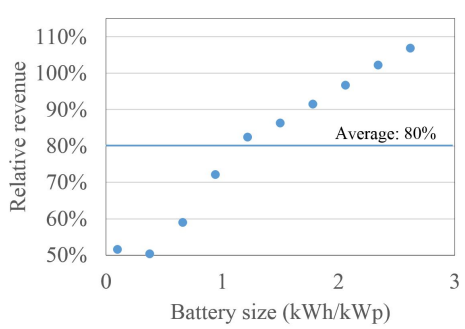

Fig. 4. Performance of the linear algorithm compared to the dynamic algorithm: (a) energy dispatch calculated for four days, with battery SOC on the top and system powers on the bottom, and (b) relative revenue between the linear and the dynamic algorithms.

(a)

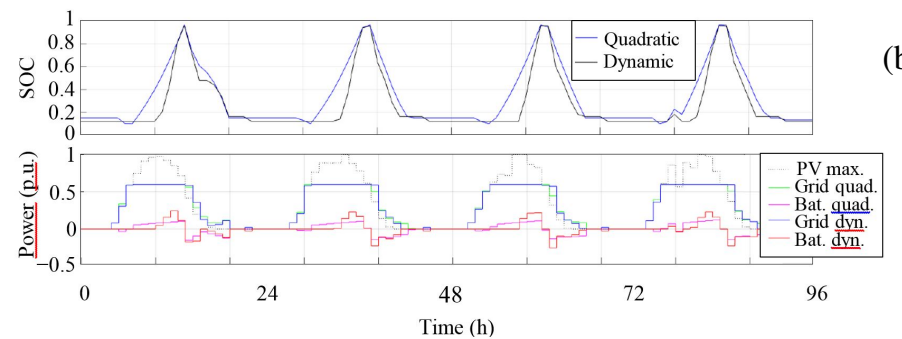

(b)

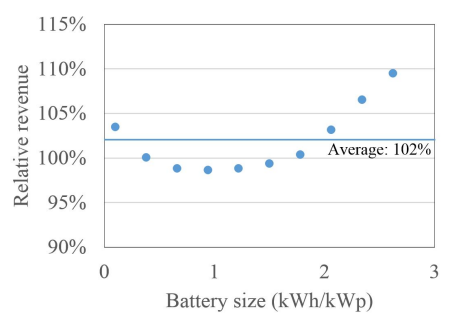

Fig. 5. Performance of the quadratic algorithm compared to the dynamic algorithm: (a) energy dispatch calculated for four days, with battery SOC on the top and system powers on the bottom, and (b) relative revenue between the quadratic and the dynamic algorithms.

explained in the previous section, that prevents the inclusion of the battery current as an input variable. However, this strategy leads to a faster battery aging, which reduces the obtained revenue. This is the reason why the relative revenue shown in Figure 4 (b) is lower than $100 \%$ for most of the battery sizes, giving an average value of $80 \%$ for the ten sizes analyzed in this contribution. The linear algorithm has a lower performance for installations with small batteries, providing a revenue as low as $50 \%$ of that obtained by a dynamic program. This is due to the repetitive power peaks scheduled by the linear model. It is noteworthy that the linear algorithm achieves better results than the dynamic for large batteries. This effect is due to the combination of two issues. On the one hand, the system is not able to manage the required power to induce fast battery charges and discharges, thereby mitigating the main drawback of the linear algorithm. On the other hand, the step size of the discrete variables required for a dynamic optimizer increases as the battery is enlarged, reducing the accuracy of the dynamic algorithm.

A summary of this comparison is provided in Table III. The second column is related to the dynamic algorithm, while the third one concerns the linear optimizer. Specially remarkable from this table is the row of computation time. The time required by a typical desktop computer to calculate the dispatch plan for a year is reduced from $2514 \mathrm{~s}$ used by the dynamic optimizer to $10.9 \mathrm{~s}$ required by the linear algorithm. This reduced complexity leads to the following row, in which the minimum time step that can be managed by each algorithm in order to solve one-day problems is provided. The shorter the time step, the larger the number of variables and, therefore, the higher the computation requirements. A linear algorithm can solve problems with a $30 \mathrm{~s}$ time step, while the dynamic algorithm is unable to solve the problem if the time step is shorter than $1 \mathrm{~h}$.

Analogously, Figure 5 shows the comparison between the dynamic and quadratic algorithms. It can be seen in Figure 5 (a) that the schedule computed by both algorithms is similar. The most notorious difference is that the quadratic algorithm schedules a slower charging process than that of the dynamic program. This leads to enhanced calendar aging, given its dependency on battery voltage, but reduced cycle aging, given its dependency on current. These two processes are similar and their overall effect is negligible, as can be seen in Figure 5 (b). The quadratic algorithm achieves a similar revenue than the dynamic optimizer for all battery sizes, being the average revenue a $102 \%$ compared to that obtained by the dynamic algorithm. Similar to the previous analysis, the relative revenue increases for large battery sizes as a result of the inaccuracy of the dynamic algorithm induced by larger discretization steps.

The forth column of Table III summarizes the main characteristics of the quadratic algorithm. It achieves a good trade-off between accuracy and simplicity. The computation time is reduced from $2514 \mathrm{~s}$ required by the dynamic program to only $13.2 \mathrm{~s}$ for a one-year simulation. Meanwhile, the obtained revenue is similar to that achieved by the dynamic program, being the average value even $2 \%$ higher. It is remarkable to note that the quadratic fit shown in Figure 2 is worse for higher current. Therefore, a battery current lower than $1.5 \mathrm{C}$ is required for a suitable performance of this algorithm. 
Table III

SUMMARY OF THE COMPARISON PRESENTED IN THIS CONTRIBUTION.

\begin{tabular}{llll}
\hline Characteristic & Dynamic & Linear & Quadratic \\
\hline $\begin{array}{l}\text { Main sources of } \\
\text { inaccuracy }\end{array}$ & $\begin{array}{l}\text { Discretization required, } \\
\text { notorious with large } \\
\text { batteries }\end{array}$ & $\begin{array}{l}\text { Cycle aging independent of power } \\
\text { Constant efficiency }\end{array}$ & $\begin{array}{l}R_{i} \text { independent of SOC } \\
\text { Joule efficiency } \\
\text { Linear fit of } V_{O C}(S O C)\end{array}$ \\
$\begin{array}{l}\text { Remarkable } \\
\text { characteristics }\end{array}$ & $\begin{array}{l}\text { Loss of optimality because } \\
\text { of discrete variables }\end{array}$ & $\begin{array}{l}\text { Higher current and faster aging } \\
\text { Poor performance for small battery }\end{array}$ & $\begin{array}{l}\text { Trade-off between accuracy } \\
\text { and computation requirements }\end{array}$ \\
Computation time & $2514 \mathrm{~s}$ & $10.9 \mathrm{~s}$ & $13.2 \mathrm{~s}$ \\
Minimum time step & $1 \mathrm{~h}$ & $30 \mathrm{~s}$ & $30 \mathrm{~s}$ \\
Max. battery current & No loss of accuracy & $0.5 \mathrm{C}$ & $1.5 \mathrm{C}$ \\
Average rel. revenue & $100 \%$ & $80 \%$ & $102 \%$ \\
Preferred & Off-line, accurate & On-line, regular processor & On-line, low range processor \\
applications & battery model & Limited to low power & Off-line, uncritical accuracy \\
\hline
\end{tabular}

\section{CONCLUSION}

This contribution presents a comparison between algorithms proposed for the energy management of a Li-ion battery in a renewable power plant. The main results obtained from the comparison between dynamic, linear and quadratic algorithms are summarized in Table III. The most notorious difference among the algorithms is the computation time. A commercial PC takes 42 minutes to compute the energy dispatch during a year by means of a dynamic algorithm, while the linear and quadratic algorithms can be run in about $10 \mathrm{~s}$. This large computation time limits the applicability of dynamic algorithms in two main aspects. Firstly, a larger number of variables entails a great increase of the problem complexity. A commercial PC is not able to solve such problem with much more than 24 variables. Therefore, each optimization problem needs to be reduced to one day, and the minimum time step that can be considered is $1 \mathrm{~h}$. This limitation is not critical for the management of PV systems, due to its daily profile, but can jeopardize the use of a dynamic algorithm for other systems, such as wind power plants. The second restriction is its limitation to discrete variables. This entails a divergence between the achieved solution and the actual optimum, which depends on the discretization step. Given that larger batteries require larger discretization steps, a dynamic algorithm achieves solutions farther from the optimum, as shown in Figures 4 (b) and 5 (b).

By contrast, the use of linear and quadratic algorithms requires a number of simplifications in the system model, objective function, and constraints that can entail a deviation in the optimization. The analysis herein presented quantifies the loss of optimality for the linear algorithm in $20 \%$. The main reason is that the current is not taken into account as an accelerator factor for the battery aging. On the other hand, the quadratic algorithm offers a trade-off between simplicity and accuracy. The average result for the analyzed scenarios overcomes the performance of the dynamic algorithm, and its computational requirements are notably lower. However, the quadratic fit presented in Figure 2 (c) shows a fit loss for high currents. Therefore, worse results are expected for the quadratic algorithm if the maximum battery current surpasses $1.5 \mathrm{C}$. Given the low current required for the renewable-energy application analyzed in this contribution, this loss of optimality is not characterized.

To sum up, this contribution establishes a tool for the selection of a proper energy management algorithm for a particular application. If accuracy is a main concern and the battery has a low energy capacity compared to the power, a dynamic algorithm may be a suitable choice. However, if the energy capacity of the battery is large compared to its maximum power, or problems with more than 24 variables need to be optimized, a quadratic algorithm may be a more clever option. Finally, linear algorithms have been proven not to be able to properly model the battery aging, and provide an average profit $20 \%$ lower than the maximum achievable.

\section{REFERENCES}

[1] T. Bunsen, P. Cazzola, M. Gorner, L. Paoli, S. Scheffer, R. Schuitmaker, J. Tattini, and J. Teter, "Global EV outlook 2018: Towards cross-modal electrification," International Energy Agency, Technical report, 2018.

[2] A. Berrueta, J. Pascual, I. San Martín, P. Sanchis, and A. Ursúa, "Influence of the aging model of lithium-ion batteries on the management of PV self-consumption systems," in EEEIC / I CPS Europe, 2018, pp. 1-5.

[3] AEMET and Gobierno de Navarra. Meteorología y climatología de Navarra. Online. [Online]. Available: http://meteo.navarra.es/estaciones/ mapadeestaciones.cfm

[4] A. Berrueta, V. Irigaray, P. Sanchis, and A. Ursúa, "Lithium-ion battery model and experimental validation," in EPE'15 ECCE-Europe, 2015.

[5] A. Berrueta, A. Urtasun, A. Ursúa, and P. Sanchis, "A comprehensive model for lithium-ion batteries: From the physical principles to an electrical model," Energy, vol. 144, pp. 286-300, 2018.

[6] A. Berrueta, M. Heck, M. Jantsch, A. Ursúa, and P. Sanchis, "Combined dynamic programming and region-elimination technique algorithm for optimal sizing and management of lithium-ion batteries for photovoltaic plants," Applied Energy, vol. 228, pp. 1 - 11, 2018.

[7] W. Waag, C. Fleischer, and D. U. Sauer, "Critical review of the methods for monitoring of lithium-ion batteries in electric and hybrid vehicles," Journal of Power Sources, vol. 258, pp. 321 - 339, 2014.

[8] J. Schmalstieg, S. Käbitz, M. Ecker, and D. U. Sauer, "A holistic aging model for li(nimnco) $\mathrm{o}_{2}$ based 18650 lithium-ion batteries," Journal of Power Sources, vol. 257, pp. 325 - 334, 2014.

[9] R. Bellman, Dynamic Programming, 1st ed. Princeton, NJ, USA: Princeton University Press, 1957. 